Pacific Journal of Mathematics

ON EXACT LOCALIZATION 


\section{ON EXACT LOCALIZATION}

\section{ROBERT A. RUBIN}

In this paper we consider certain aspects of exact localization (idempotent kernel functors having Property $(T)$ in the language we shall be employing). The major result is that for commutative noetherian rings, every idempotent kernel functor has Property $(T)$ if and only if the Krull dimension of the ring is less than or equal to 1.

1. Preliminaries. The terminology and notation in this paper are that of Goldman [1], with which familiarity is assumed. In particular, if $\Lambda$ is a ring we denote by $K(\Lambda)$ (respectively $I(\Lambda)$ ) the set of kernel functors (respectively idempotent kernel functors on the category of left $\Lambda$-modules) belonging to $\Lambda$. If $\sigma \in K(\Lambda)$, we denote by $\mathscr{T}_{\sigma}$ the associated filter of left ideals; i.e., $\mathscr{T}_{\sigma}$ is the set of left ideals $\mathfrak{X}$ of $\Lambda$ such that $\Lambda / \mathfrak{A}$ is $\sigma$-torsion, and such an $\mathfrak{A}$ is called a $\sigma$-open left ideal. Finally by the term "module" we mean a left module over the ring in question.

Our primary concern will be with kernel functors satisfying any of the conditions of Theorem 4.3 of [1], which we restate for easy reference.

THEOREM 1.1. For any $\sigma \in I(\Lambda)$, the following conditions are equivalent:

(i ) $Q_{\sigma}(M) \approx Q_{\sigma}(\Lambda) \bigotimes_{\Lambda} M$ for every module $M$;

(ii) $Q_{\sigma}(\Lambda) i(\mathfrak{U})=Q_{\sigma}(\Lambda)$ for every $\mathfrak{X} \in \mathscr{I}_{\sigma^{\prime}}$ where $i$ is the canonical $\operatorname{map} \Lambda \rightarrow Q_{\sigma}(\Lambda)$;

(iii) Every $Q_{\sigma}(\Lambda)$-module is faithfully $\sigma$-injective as a $\Lambda$-module; i.e., given a $Q_{\sigma}(\Lambda)$-module $X$, $\Lambda$-modules $B \cong A$ with $\sigma(A / B)=A / B$ and a A-homomorphism $f: B \rightarrow X$, there is a unique A-homomorphsim $g$ : $A \rightarrow X$ extending $f$;

(iv) Every $Q_{\sigma}(\Lambda)$-module is $\sigma$-torsion-free as a $\Lambda$-module;

(v) The functor $Q_{\sigma}$ is right exact and commutes with direct sums.

An idempotent kernel functor satisfying any of the above conditions is said to have Property (T). Each of the conditions in (v) above has a useful equivalent (Theorems 4.3 and 4.4 of [1]) which we also list.

THEOREM 1.2. For $\sigma \in I(\Lambda)$, the following are equivalent:

(i) $Q_{\sigma}$ is a right exact functor;

(ii) If $\mathfrak{A} \in \mathscr{T}_{\sigma}$, if $M \stackrel{\pi}{\longrightarrow} M^{\prime \prime}$ is an epimorphism of $\sigma$-torsion-free modules, and if $f: \mathfrak{A} \rightarrow M^{\prime \prime}$ is a homomorphism, then there exists $\mathfrak{B} \in$ 
$\mathscr{T}_{\sigma}$ with $\mathfrak{B} \subseteq \mathfrak{A}$ and $g: \mathfrak{B} \rightarrow M$ such that $\pi g$ is the restriction of $f$ to $\mathfrak{B}$.

THeOREM 1.3. For $\sigma \in I(\Lambda)$ the following are equivalent:

(i) $Q_{\sigma}$ commutes with direct sums;

(ii) Given any countable chain of left ideals whose union is $\sigma$ open, then some member of the chain is $\sigma$-open.

REMARKs. (i) The condition of Theorem 1.2 (ii) is described by saying that every $\sigma$-open left ideal is $\sigma$-projective, while that of Theorem 1.3 (ii) is given by: $\sigma$ is noetherian.

(ii) Since for $\sigma$ with Property $(T)$ every $\sigma$-open left ideal contains a finitely generated $\sigma$-open left ideal, it has been asked whether, for $\sigma$ noetherian, every $\sigma$-open contains a finitely generated $\sigma$-open. We give an example, due to G. Bergman, that supplies the negative answer. Let $G$ be a nondiscrete ordered group in which the identity $e$ is not the inf of any countable set of elements greater than $e$ (e.g., let $G$ be an uncountable product of the integers ordered lexicographically). Let $K$ be a field and let $S=\{g \in G \mid g>e\}$. If $R=K[S]$, the semigroup algebra of $S$ over $K$, let $I$ be the ideal of $R$ generated by all $g \in S$. Then $I$ is a maximal ideal and $I^{2}=I$. Hence $\mathscr{T}=\{I, R\}$ defines $\mu \in I(R)$. Let $J_{1} \leqq J_{2} \leqq \cdots$ be a countable chain of ideals with $J_{i} \neq I, R$ for each $i$. Then for each $i$ there is $g_{i} \in S$ with $e \neq g_{i} \notin J_{i}$. Since $e \neq \inf g_{i}$, there is $g \in S$ with $e \neq g \leqq g_{i}$ for each $i$. Then $g \notin \cup J_{i}$, and so $\cup J_{i} \notin \mathscr{T}$. Thus $\mu$ is noetherian, but $I$ does not contain a finitely generated $\mu$-open ideal.

In considering Property $(T)$ we shall at times make simplifying assumptions that entail no loss of generality. The first such is that the ring be torsion-free for the kernel functor under investigation.

Let $\sigma \in K(\Lambda)$, and consider $\left\{\mathfrak{N} / \sigma(\Lambda) \mid \mathfrak{A} \in \mathscr{T}_{\sigma}\right.$ and $\left.\mathfrak{X} \supseteqq \sigma(\Lambda)\right\}$. It is routine to check that this defines a kernel functor of $\Lambda / \sigma(\Lambda)$, which we denote by $\sigma_{*}$, and that $\sigma_{*}$ is idempotent if $\sigma$ is.

Proposition 1.4. Let $\sigma \in I(\Lambda)$ and let $\sigma_{*} \in I(\Lambda / \sigma(\Lambda))$ be defined as above. Then $\sigma$ has Property $(T)$ if and only if $\sigma_{*}$ has Property $(T)$.

Proof. It is immediate that if $\sigma$ is noetherian, so is $\sigma_{*}$. Using the fact that for $\sigma$ idempotent, $\mathfrak{A}+\sigma(\Lambda) \sigma$-open implies $\mathfrak{A}$ is $\sigma$-open we see that if $\sigma_{*}$ is noetherian, so is $\sigma$.

Now since $\sigma$-torsion-free $\Lambda$-modules may be identified with $\sigma_{*}$ torsion-free $\Lambda / \sigma(\Lambda)$-modules, and since whenever we have $\mathfrak{A} \stackrel{f}{\longrightarrow} M$ with $M \sigma$-torsion-free, $f$ factors through $\mathfrak{U}+\sigma(\Lambda) / \sigma(\Lambda) \approx \mathfrak{X} / \mathfrak{A} \cap \sigma(\Lambda)$ we see that every $\sigma$-open left ideal of $\Lambda$ being $\sigma$-projective is equivalent to every $\sigma_{*}$-open left ideal of $\Lambda / \sigma(\Lambda)$ being $\sigma_{*}$-projective. Thus Theorems 


\section{$1.1(\mathrm{v}), 1.2$, and 1.3 give the result.}

We now consider certain classes of rings for which it is possible to decide, using familiar concepts, when every idempotent kernel functor has Property $(T)$. The first such class we shall consider is the class of rings for which every idempotent kernel functor has a minimal open ideal.

LEMma 1.5. Let $\sigma \in I(\Lambda)$ be such that $\mathscr{T}_{\sigma}$ has minimal elements. Then there is a unique idempotent two-sided ideal, $L_{\sigma}$, such that $\mathscr{T}_{\sigma}=$ $\left\{\mathfrak{A} \subseteq \Lambda \mid \mathfrak{A} \supseteqq L_{\sigma}\right\}$.

Proof. Since $\mathscr{T}_{\sigma}$ is closed under intersections, it has a unique minimal element, a left ideal we denote by $L_{\sigma}$. Now the uniqueness and minimality of $L_{\sigma}$ guarantee that $\mathscr{T}_{\sigma}=\left\{\mathfrak{X} \cong \Lambda \mid \mathfrak{X} \supseteqq L_{\sigma}\right\}$. Furthermore, $\mathscr{T}_{\sigma}$ is closed under residual division, whence $L_{\sigma}$ is two-sided, and under products of left ideals, from which it follows that $L_{\sigma}$ is idempotent.

THEOREM 1.6. Let $\sigma \in I(\Lambda)$ be noetherian, and suppose that $\mathscr{T}_{\sigma}$ has a unique minimal element $L_{\sigma}$. Then $\sigma$ has Property $(T)$ if and only if $L_{\sigma}+\sigma(\Lambda) / \sigma(\Lambda)$ is a projective $\Lambda / \sigma(\Lambda)$ ideal.

Proof. Form $\sigma_{*} \in I(\Lambda / \sigma(\Lambda))$ as for Prop. 1.4. Then $\mathscr{T}_{\sigma *}$ has unique minimal element $L_{\sigma}+\sigma(\Lambda) / \sigma(\Lambda)$. Hence by Prop. 1.4 we may assume that $\sigma(\Lambda)=0$. Suppose now that $\sigma$ has Property $(T)$. Let $F$ be a free module mapping onto $L_{o}$ by $p$. Since $\sigma(\Lambda)=0, \sigma(F)=$ $\sigma\left(L_{\sigma}\right)=0$. Thus by Theorems $1.1(\mathrm{v})$ and 1.2 the identity map on $L_{o}$ splits $p$, and $L_{\sigma}$ is projective. Conversely, suppose that $L_{\sigma}$ is a projective ideal. Then clearly the condition of Theorem 1.2 (ii) holds, and together with the noetherian hypothesis, this implies that $\sigma$ has Property $(T)$.

REMARK. The example following Theorem 1.3 gives an example of an idempotent noetherian kernel functor whose filter has a unique minimal element, yet which fails to have Property $(T)$.

CoROLlaRY 1.7. Let $\Lambda$ be left artinian, and suppose that every idempotent two-sided ideal of $\Lambda$ is projective. Then every $\sigma \in I(\Lambda)$ has Property (T).

Proof. If $L$ is a projective ideal of $\Lambda$, then for any ideal $I$, $(L+I) / I$ is a projective ideal of $\Lambda / I$.

Corollary 1.8. Let $R$ be a commutative artinian ring. Then 
every $\sigma \in I(R)$ has Property $(T)$.

We now turn to the class of commutative noetherian rings. The crucial fact for these rings is that Property $(T)$ is a local property.

If $R$ is a commutative ring and $X$ a multiplicative subset of $R$, we define $\mu_{x}$ via: if $M$ is an $R$-module, $\mu_{X}(M)=\{m \in M \mid x m=0$ for some $x \in X\}$. Then $\mu_{X} \in I(R)$, an ideal $A$ of $R$ is $\mu_{X}$-open if and only if $A \cap X \neq \varnothing$, and for any module $M, Q_{\mu_{X}}(M)=M_{X}$ (or $\left.X^{-1} M\right)$. As the next lemma shows, we may also localize kernel functors.

LEMma 1.9. Let $R$ be a commutative ring, $X$ a multiplicative subset of $R$, and $\rho \in I(R)$. Then $\left\{A_{X} \mid A \in \mathscr{T}_{\rho}\right\}=\mathscr{F}$ defines an idempotent kernel functor of $R_{X}$, which we denote by $\rho_{X}$.

Proof. That $\mathscr{F}$ defines a kernel functor, $\rho_{X}$, of $R_{X}$ is routine, following from the extension and contraction properties of ideals under localization. (See [5] p. 46, for instance.) For an ideal $K$ of $R_{X}$ let $K \cap R$ denote the inverse image of $K$ under the canonical map from $R$ to $R_{X}$. Now if $K \subseteq L$ are ideals of $R_{X}$ with $L \rho_{X}$-open, and $L / K$ $\rho_{X}$-torsion, let $A=L \cap R$ and $B=K \cap R$. Then $A$ is $\rho$-open, and $A / B$ is $\rho$-torsion. Hence by [1], Theorem 2.5, $B$ is $\rho$-open. But $K=B_{X}$, so $K$ is $\rho_{X}$-open. Then [1], Theorem 2.5 applies again to show that $\rho_{X}$ is idempotent.

Proposition 1.10. Let $R$ be a commutative ring, $X$ a multiplicative subset of $R$, and $\rho \in I(R)$ an idempotent kernel functor such that every o-open ideal contains a finitely generated $\rho$-open ideal. Then for any $R$-module $M,\left[Q_{\rho}(M)\right]_{X}=Q_{\rho_{X}}\left(M_{X}\right)$.

Proof. Since an easy calculation shows that $(\rho(M))_{X}=\rho_{X}\left(M_{X}\right)$ (the finiteness condition is needed here) we may assume that $\rho(M)=$ $\rho_{X}\left(M_{X}\right)=0$. Then it is well-known that $Q_{\rho}(M)={\underset{\lim }{A \in \sigma_{\rho}}}_{\operatorname{Hom}_{R}}(A, M)$, and we may take the $A$ 's to be finitely generated. (See [3] p. 11 for instance.) But

$$
\begin{aligned}
\left(Q_{\rho}(M)\right)_{X} & \cong R_{X} \bigotimes_{R} \stackrel{\lim }{A}_{\longrightarrow} \operatorname{Hom}_{R}(A, M) \cong \lim _{A}\left[R_{X} \bigotimes_{R} \operatorname{Hom}_{R}(A, M)\right] \\
& \cong \lim _{A}\left[\operatorname{Hom}_{R_{X}}\left(A_{X}, M_{X}\right)\right] \cong Q_{\rho_{X}}\left(M_{X}\right),
\end{aligned}
$$

since surely this limit is the same as that taken over $A_{X}$. (We have used here the commonly known facts that tensor product commutes with direct limits and Hom commutes with localization when the domain is finitely generated.)

As usual, when $P$ is a prime ideal of $R$, we shall write $\rho_{P}$ instead 
of $\rho_{R-P}$.

THEOREM 1.11. Let $R$ be a commutative noetherian ring, and let $\sigma \in I(R)$. Then $\sigma$ has Property $(T)$ if and only if for every prime ideal $P$ of $R, \sigma_{P} \in I\left(R_{P}\right)$ has Property $(T)$.

Proof. Suppose $\sigma$ has Property $(T)$, and let $V$ be an $R_{P}$-module. Then $Q_{\sigma_{P}}\left(R_{P}\right) \bigotimes_{R_{P}} V \cong\left[Q_{\sigma}(R)\right]_{P} \bigotimes_{R_{P}} V \cong\left(Q_{\sigma}(R) \bigotimes_{R} V\right)_{P} \cong\left(Q_{\sigma}(V)\right)_{P} \cong$ $Q_{o_{P}}(V)$. Thus $\sigma_{P}$ has Property $(T)$. Conversely, suppose that for every prime ideal $P, \sigma_{P}$ has Property $(T)$, and let $M$ be an $R$-module. Then there is a homomorphism $\alpha: Q_{\sigma}(R) \bigotimes_{R} M \rightarrow Q_{\sigma}(M)$ (this is just the statement that $Q_{\sigma}(M)$ is a $Q_{\sigma}(R)$-module). Then for any prime ideal $P, \alpha_{P}:\left(Q_{\sigma}(R) \bigotimes_{R} M\right)_{P} \rightarrow Q_{\sigma}(M)_{P}$. But

$$
\left(Q_{\sigma}(R) \bigotimes_{R} M\right)_{P} \cong Q_{\sigma P}(R) \bigotimes_{R_{P}} M_{P}
$$

and $Q_{\sigma}(M)_{P} \cong Q_{\sigma_{P}}\left(M_{P}\right)$ by Prop. 1.10. Since $\sigma_{P}$ has Property $(T)$ for every $P, \alpha_{P}$ is an isomorphism for every $P$, and so $\alpha$ is an isomorphism.

REMARK. An examination of the proof yields that $\sigma$ has Property $(T)$ if and only if $\sigma_{m}$ has Property $(T)$ for all maximal ideals $m$. Now if $P$ is a prime ideal of $R$ and $P \notin \mathscr{I}_{o}$, then $\mathfrak{A} \in \mathscr{T}_{\sigma}$ implies $\mathfrak{A} \nsubseteq P$, and so every $\sigma$-open ideal meets $R-P$. Hence $\sigma_{p}=0$; i.e., $\mathscr{T}_{\sigma_{p}}=$ $\left\{R_{p}\right\}$. Thus $\sigma$ has Property $(T)$ if and only if $\sigma_{m}$ has Property $(T)$ for every maximal ideal $m \in \mathscr{T}_{\sigma}$.

We are now prepared to investigate the condition that every $\sigma \epsilon$ $I(R)$ has Property $(T)$.

Recall that if $R$ is a commutative ring, and $P$ a prime ideal of $R$, the height of $P, h t(P)$, is defined to be the sup of the length of chains of prime ideals: $P_{0} \subset P_{1} \subset \cdots \subset P_{r}=P$. Furthermore, if $I$ is an ideal of $R, h t(I)=\inf \{h t(P) \mid P$ is a prime ideal containing $I\}$. It will be convenient for our purposes to declare $h t(R)=\infty$.

NoTE. When we are dealing with integral domains, by the term "minimal prime ideal" we mean a prime ideal minimal among the collection of all nonzero prime ideals. Thus for integral domains a minimal prime ideal is the same thing as a height 1 prime ideal.

LEMMA 1.12. Let $R$ be an integral domain, with quotient field $k$, such that $R=\bigcap_{p} R_{p}$, where $P$ runs through some collection of prime ideals all with height $\leqq$ some fixed integer $n$. If I is an ideal of height $>n$, then $I^{-1}=R$, where $I^{-1}=\{\alpha \in k \mid \alpha I \leqq R\}$.

Proof. Let $\alpha \in I^{-1}$ and let $Q$ be any prime ideal of height $\leqq n$. Now $\alpha I \subseteq R$, and since $h t(I)>n, I \not \subset Q$. So there is $x \in I$ with $x \notin Q$. 
But $\alpha x \in R \subseteq R_{Q^{\prime}}$ and since $x \notin Q, x$ is a unit in $R_{Q}$. Thus $\alpha=(\alpha x) x^{-1} \in$ $R_{Q}$, and $\alpha$ is in $R_{Q}$ for every prime ideal of height $\leqq n$. Since $R$ is an intersection of such rings, $\alpha \in R$. So $I^{-1} \subseteq R$. But certainly $R \subseteq$ $I^{-1}$ and we are done.

LEMMA 1.13. Let $R$ be a commutative ring, $n$ a nonnegative integer, and $\mathscr{F}=\{I \subseteq R \mid h t(I)>n\}$. Then defines an idempotent kernel functor; i.e., there is $\sigma \in I(R)$ such that $\mathscr{F}=\mathscr{T}_{\sigma}$.

Proof. $H t(I)>n$ means the same thing as $I$ is contained in no prime ideal of height $\leqq n$. Thus we have:

(i) if $I \in \mathscr{F}$, and $J \supseteqq I$, then $J$ cannot be contained in any prime ideal of height $\leqq n$, for then $I$ would as well; so $J \in \mathscr{F}$;

(ii) if $I, J \in \mathscr{F}$ and $P$ is a prime ideal of $h t \leqq n$, then $I J \subseteq P$ implies $I \subseteq P$ or $J \subseteq P$, a contradiction; thus $I J$, and so $I \cap J, \in \mathscr{F}$;

(iii) let $I \in \mathscr{F}$ and $J \subseteq I$ be such that for any $x \in I$ there is a $K \in \mathscr{F}$ with $K x \leqq J$, and suppose that $J \subseteq P$ for some prime ideal $P$ with $h t(P) \leqq n$. Now $I \not \subset P$, so there is $x \in I$ with $x \notin P$. But there is $K \in \mathscr{F}$ with $K x \subseteq J \subseteq P$, and so $K \subseteq P$, contradicting $h t(K)>$ $n$. Thus $J \in \mathscr{F}$. Hence $\mathscr{F}$ defines an idempotent kernel functor.

Recall that an integral domain $R$ is called a Krull domain if (i) $R=\bigcap_{p} R_{p^{\prime}}$ where $P$ runs through the minimal prime ideals;

(ii) for each minimal prime ideal $P, R_{p}$ is a discrete valuation ring; and (iii) if $\alpha \in k$, the quotient field of $R, \alpha$ is a unit in all but finitely many of the $R_{p}, P$ a minimal prime ideal. See [2, 4] for more about Krull domains.

In the following theorem we shall have need of the following well-known fact (see [2] Ex. 2. p. 83 for instance): an integral domain $R$ is a Dedekind domain if and only if $R$ is a Krull domain and every nonzero prime ideal of $R$ is maximal.

THeOREM 1.14. Let $R$ be a Krull domain. Then every $\sigma \in I(R)$ has Property $(T)$ if and only if $R$ is a Dedekind domain.

Proof. Suppose that every $\sigma \in I(R)$ has Property $(T)$, and let $\mu \in I(R)$ be defined by $\mathscr{T}_{\mu}=\{I \subseteq R \mid h t(I)>1\}$. Now for any integral domain $D$ and any $\rho \in I(D), Q_{\rho}(D)=\bigcup_{J \in \mathscr{T}_{\rho}} J^{-1}$. So $Q_{\mu}(R)=\mathrm{U}_{I \in \mathscr{F}_{\mu}} I^{-1}$. But by Lemma 1.12, for each $I \in \mathscr{T}_{\mu}, I^{-1}=R$, and so $Q_{\mu}(R)=R$. But $\mu$ has Property $(T)$, and so $Q_{\mu}(R) I=Q_{\mu}(R)$ for any $I \in \mathscr{T}_{\mu}$; i.e., for any $I \in \mathscr{T}_{\mu}, R I=R$. Thus $\mathscr{T}_{\mu}=\{R\}$, and the only ideal of height $>1$ is $R$ itself. Hence every nonzero prime is of height 1; i.e., every nonzero prime ideal of $R$ is maximal, as so by the remark preceding the theorem, $R$ is Dedekind. 
Conversely, if $R$ is Dedekind, $R$ is an hereditary noetherian ring, and be the discussion on page 31 of [1] every $\sigma \in I(R)$ has Property $(T)$.

THEOREM 1.15. Let $R$ be a commutative noetherian integral domain. Then every $\sigma \in I(R)$ has Property $(T)$ if and only if every nonzero prime ideal of $R$ is maximal; (i.e., Krull dimension of $R \leqq 1$ ).

Proof. Suppose that every nonzero prime ideal of $R$ is maximal. Since $R$ is noetherian, Theorem 1.11 holds, and we may assume that $R$ is local. If $R$ is a field, $I(R)=\{0, \infty\}$, both of which have Property $(T)$, and we are done. So suppose that $R$ is not a field. Then the set of nonzero ideals defines $\mu \in I(R)$, and since $\mu=\mu_{X}$, where $X$ is the set of nonzero elements of $R, \mu$ has Property $(T)$. Now let $\sigma \in I(R), \sigma \neq 0, \infty$. Then $0 \notin \mathscr{T}_{\sigma}$, and for some $\mathfrak{A} \neq R, \mathfrak{N} \in \mathscr{T}_{\sigma}$. Since $\mathfrak{U} \subseteq \mathscr{M}$ and $\sigma$ is idempotent, $\mathscr{M}^{n} \in \mathscr{T}_{\sigma}$ for any $n \geqq 0$. Let $J$ be any nonzero ideal of $R$. Since $R$ is noetherian, $J$ contains a power of its radical which, due to a lack of other candidates, is $\mathscr{L l}$. Thus $J \supseteqq \mathscr{C}^{n}$ for some $n$, and so $J \in \mathscr{T}_{\sigma}$. Hence $\sigma=\mu$, and so every $\sigma \in I(R)$ has Property $(T)$.

Conversely suppose that every $\sigma \in I(R)$ has Property $(T)$. Again by Theorem 1.11 we may assume that $R$ is local (with maximal ideal $\mathscr{C}$ ), for the condition that every nonzero prime is maximal is itself a local property. Let $S$ be the integral closure of $R$ in its quotient field $k$. Then $S$ is a Krull domain (p. 82 of [2]). Define $\sigma \in I(R)$ by $\mathscr{T}_{\sigma}=\left\{\mathfrak{A} \subseteq R \mid \mathfrak{A} \supseteq \mathscr{C}^{n}\right.$ for some $\left.n\right\}$. Then $Q_{\sigma}(R)=\mathrm{U}_{n} \mathscr{C}^{-n}$, where $\mathscr{M}^{-n}=\left(\mathscr{M}^{n}\right)^{-1}=\left\{\alpha \in k \mid \alpha \mathscr{K}^{n} \subseteq R\right\}\left(Q_{o}(R)\right.$ is also known as the ideal transform of $\mathscr{C}$ ). Consider the set $\mathfrak{X}$ of prime ideals of $S$ that contain $\overline{\mathscr{C}}=\mathscr{M} S$. By Theorem 33.10 of [4], $\mathfrak{X}$ is finite, and by Theorem 44 p. 29 of [2], $\mathfrak{X}$ consists entirely of maximal ideal of $S$. Write $\mathfrak{X}=\left\{\mathscr{N}_{1}, \cdots, \mathscr{N}_{l}, P_{1}, \cdots, P_{r}\right\}$ where all the ideals are distinct and the $P_{j}$ 's are all the minimal ideals of $S$ that contain $\overline{\mathscr{l}}$. Since $S$ is Krull, each $S_{p_{j}}$ is a discrete valuation ring, and so $\bigcap_{k} P_{j}^{k}=0$ for each $j$. So for each $j=1, \cdots, r$ there is $n_{j} \geqq 1$ such that $\overline{\mathscr{K}} \leqq$ $P_{j}^{n_{j}}$ but $\overline{\mathscr{C}} \nsubseteq P_{j}^{n_{j}+1}$. Since the $P_{j}$ 's are distinct maximal ideals, $\Pi_{j=1}^{r} P_{j}^{n_{j}}=\bigcap_{j=1}^{r} P_{j}^{n_{j}} \supseteqq \overline{\mathscr{C}}$. Set $A=\prod_{j=1}^{r} P_{j}^{n_{j}}$. Now by Exercise 7, p. 83 of [2], if $P$ is a minimal prime ideal in a Krull domain, $P P^{-1} \supsetneq P$. Hence for each $j=1, \cdots, r, P_{j}$ is invertible (i.e., $P_{j} P_{j}^{-1}=S$ ). So $A$ is invertible and $A A^{-1}=S$. Consequently $\overline{\mathscr{M}}=S \overline{\mathscr{M}}=\left(A A^{-1}\right) \mathscr{M}=$ $A\left(A^{-1} \overline{\mathscr{M}}\right)$. Let $B=A^{-1} \overline{\mathscr{C}}$. Then since $\overline{\mathscr{M}} \sqsubseteq A, B \leqq S$, and $B A=$ $\overline{\mathscr{K}}$. Furthermore since $A^{-1} \supseteqq S, B \supseteqq \overline{\mathscr{C}}$. Let $P$ be a height 1 prime ideal of $S$ and suppose that $B \cong P$. Then $\overline{\mathscr{C}} \cong P^{n_{j_{0}+1}}$ so that $P=P_{j_{0}}$ for some $j_{0}$, and $\overline{\mathscr{M}}=B A \subseteq P_{j_{0}}\left(\Pi_{j=1}^{r} P_{j}^{n} j\right) \subseteq P_{j_{0}}$, a contradiction. Thus $h t(B)>1$, and by Lemma $1.12, B^{-1}=S$. Similarly $B^{-n}=$ $S$ for all $n \geqq 1$. Now $B A \leqq A$, so that $(B A)^{-1} \supseteqq A^{-1}$. Let $\alpha \in(B A)^{-1}$. 
Then $\alpha B A \subseteq S$, and for any $x \in A, \alpha x B \subseteq S$. So $\alpha A \subseteq B^{-1}=S$, and hence $A^{-1}=(B A)^{-1}$. Thus $\overline{\mathscr{C}}^{-1}=(B A)^{-1}=A^{-1}$, and similarly, for any $n \geqq 1, \overline{\mathscr{C}}^{-n}=A^{-n}$. Now suppose that $\sigma$ has Property $(T)$. Then by Theorem 1.1 (ii) $\mathscr{M} Q_{\sigma}(R)=Q_{\sigma}(R)$, and so for some $t, 1 \in \mathscr{M} \mathscr{M}^{-t}$. But $\mathscr{M}^{-t} \leqq \overline{\mathscr{K}}^{-t}$, and so $1 \in \overline{\mathscr{M}} \overline{\mathscr{M}}^{-t}=\overline{\mathscr{M}}\left(A^{-t}\right)$. Since $S \subseteq \overline{\mathscr{M}} A^{-t}$ and $A$ is invertible, $A^{t} \subseteq \overline{\mathscr{C}}$. Consequently there are no $\mathscr{N}_{i}$ 's in $\mathfrak{X}$. Thus $\overline{\mathscr{M}}$ is contained only in minimal ideals of $S$. But then Theorem 44 p. 29 of [2] tells us that $\mathscr{M}$ is a minimal prime ideal of $R$, and the proof is complete.

The preceding theorem is the means by which we can determine when every idempotent kernel functor has Property $(T)$ for an arbitrary commutative noetherian ring. The following lemma is rather interesting by itself. Recall that if $P$ is a prime ideal in a commutative $R$, then $\mu_{p} \in I(R)$ is given by $\mathscr{T}_{\mu_{p}}=\{I \leqq R \mid I \nsubseteq P\}$, and $Q_{\mu_{p}}(M)=$ $M_{p} \approx R_{p} \bigotimes_{R} M$ for any module $M$.

Lemma 1.16. Let $R$ be a commutative ring, and let $P_{1}, \cdots, P_{n}$ be prime ideals of $R$. Then $\inf _{i} \mu_{p_{i}}=\mu_{X}$, where $X=\bigcap_{i=1}^{n}\left(R-P_{i}\right)$ and $\inf _{\alpha} \sigma_{\alpha}$ is defined by $\left(\inf _{\alpha} \sigma_{\alpha}\right) M=\bigcap_{\alpha} \sigma_{\alpha}(M) M$ any module, or equivalently $\mathscr{T}_{\mathrm{inf} \sigma_{\alpha}}=\bigcap_{\alpha} \mathscr{T}_{\sigma_{\alpha}}$, for any $\left\{\sigma_{\alpha}\right\} \leqq K(R)$.

Proof. Let $\sigma=\inf _{i} \mu_{p_{i}}$. If $\mathfrak{N} \in \mathscr{T}_{\sigma}$, then $\mathfrak{A} \in \mathscr{T}_{\mu_{p_{i}}}$ for each $i=$ $1, \cdots, n$, and so $\mathfrak{A} \nsubseteq P_{i}$ for each $i$. But then $\mathfrak{A} \nsubseteq \cup P_{i}$ (see Theorem 81 p. 55 of [2] for instance). So there is $a \in \mathfrak{N}$ such that $a \in \bigcap_{i=1}^{n}(R-$ $P_{i}$ ) thus $\mathfrak{X} \cap X \neq \varnothing$, and $\sigma \leqq \mu_{X}$. But if $\mathfrak{B} \in \mathscr{T}_{\mu_{X}}$, there is $b \in \mathfrak{B}$ with $b \notin P_{i}$ for each $i$. Then certainly $\mathfrak{b} \nsubseteq P_{i}$ for each $i$, so that $\mu_{X} \leqq \sigma$. Hence $\inf _{i} \mu_{p_{i}}=\mu_{X}$.

CoRollary 1.17. If $R$ is a commutative ring and $P_{i}, \cdots, P_{n}$ are prime ideals, then $\inf _{i} \mu_{p_{i}}$ has Property $(T)$.

THEOREM 1.18. Let $R$ be a commutative noetherian ring. Then every $\sigma \in I(R)$ has Property $(T)$ if and only if every nonminimal prime ideal of $R$ is maximal (i.e., if $P$ is a prime ideal of $R, h t P \leqq 1$ ).

Proof. Since both of the conditions are local properties we may assume at the outset that $R$ is local, with maximal ideal $\mathscr{M}$. Suppose that every nonminimal prime ideal is maximal. Since $R$ is noetherian, a primary decomposition of 0 exists, from which we conclude that $R$ has only finitely many minimal prime ideals. Thus the set of prime ideals of $R$ consists of $\mathscr{C}$ and finitely many minimal primes $P_{1}, \cdots, P_{n}$. Now $\mu_{\mathscr{M}}=0$ (i.e., $\mathscr{T}_{\mu_{\mathscr{N}}}=\{R\}$ ), and if $0, \infty \neq \sigma \in I(R)$, then by the discussion on page 34 of [1], $\sigma=\inf \left\{\mu_{p_{i_{1}}}, \cdots, \mu_{p_{i}}\right\}$, which, by Corollary 1.17, has Property $(T)$. Since 0 and $\infty$ both have Property $(T)$, all $\sigma \in$ 
$I(R)$ have Property $(T)$. Conversely suppose that every $\sigma \in I(R)$ has Property $(T)$ and let $P \neq \mathscr{C}$ be a prime ideal of $R$. Then the ring $R / P$ is a local noetherian integral domain. Furthermore, it is easy to check that: $K(R) \rightarrow K(R / P)$, induced by $R \rightarrow R / P$, is onto. Then using Theorem 1.1 (iv) one can show that every $\rho \in I(R / P)$ has Property $(T)$, and so by Theorem $1.15, \mathscr{C} / P$ is the only nonzero prime ideal of $R / P$. Hence $P$ is a minimal prime ideal of $R$, and the proof is complete.

Finally we give an example to show that the noetherian hypothesis is essential in the preceding theorems. Let $X$ be the following semigroup: as a set, $X=\left\{x^{\alpha} \mid \alpha\right.$ is a positive real number $\}$, and $x^{\alpha} \cdot x^{\beta}=$ $x^{\alpha+\beta}$. If $K$ is a field, let $S=K[X]$, the semigroup algebra of $X$ on $K$. Then $\mathscr{C}$, the ideal generated by all the $x^{\alpha}$ (i.e., the ideal consisting of elements with no constant term), is a maximal ideal of $S$. Let $R=S_{\mathscr{N}}$. It is routine to check that the ideals of $R$ are linearly ordered and that $\mathscr{C} R$ and 0 are the only prime ideals of $R$. Furthemore $(\mathscr{L} R)^{2}=\mathscr{M} R$. Thus $\{\mathscr{L} R, R\}$ defines some $\sigma \in I(R)$. But since $\mathscr{C} R$ is not finitely generated $\sigma$ cannot have Property $(T)$. Thus $R$ is an integral domain for which every nonzero prime ideal is maximal, yet not every idempotent kernel functor has Property $(T)$.

The author wishes to express his gratitude to Paul Eakin for his generous expenditure of time and information.

\section{REFERENCES}

1. O. Goldman, Rings and modules of quotients, J. Algebra, 13 (1969), 10-47.

2. I. Kaplansky, Commutative Algebra, Allyn \& Bacon, Inc., Boston, 1970.

3. J. Lambek, Torsion Theories, Additive Semantics, and Rings of Quotients, Springer Lecture Notes No. 177, 1971.

4. M. Nagata, Local Rings, Interscience, New York, 1962.

5. D. G. Northcott, Ideal Theory, Cambridge University Press, 1953.

Received August 14, 1972 and in revised form July 17, 1973.

UNIVERSITY OF KENTUCKY

AND

Pahlavi University, Shiraz, Iran 



\section{PACIFIC JOURNAL OF MATHEMATICS}

\section{EDITORS}

RICHARD ARENS (Managing Editor)

University of California

Los Angeles, California 90024

\section{R. A. Beaumont}

University of Washington

Seattle, Washington 98105

\section{J. DuGundJI*}

Department of Mathematics

University of Southern California

Los Angeles, California 90007

D. Gilbarg and J. Milgram

Stanford University

Stanford, California 94305

ASSOCIATE EDITORS
E. F. BECKENBACH
B. H. NeumanN
F. WOLF
K. YoSHIDA

\section{SUPPORTING INSTITUTIONS}

UNIVERSITY OF BRITISH COLUMBIA

CALIFORNIA INSTITUTE OF TECHNOLOGY

UNIVERSITY OF CALIFORNIA

MONTANA STATE UNIVERSITY

UNIVERSITY OF NEVADA

NEW MEXICO STATE UNIVERSITY

OREGON STATE UNIVERSITY

UNIVERSITY OF OREGON

OSAKA UNIVERSITY

\author{
UNIVERSITY OF SOUTHERN CALIFORNIA \\ STANFORD UNIVERSITY \\ UNIVERSITY OF TOKYO \\ UNIVERSITY OF UTAH \\ WASHINGTON STATE UNIVERSITY \\ UNIVERSITY OF WASHINGTON \\ AMERICAN MATHEMATICAL SOCIETY \\ NAVAL WEAPONS CENTER
}

The Supporting Institutions listed above contribute to the cost of publication of this Journal, but they are not owners or publishers and have no responsibility for its content or policies.

Mathematical papers intended for publication in the Pacific Journal of Mathematics should be in typed form or offset-reproduced, (not dittoed), double spaced with large margins. Underline Greek letters in red, German in green, and script in blue. The first paragraph or two must be capable of being used separately as a synopsis of the entire paper. Items of the bibliography should not be cited there unless absolutely necessary, in which case they must be identified by author and Journal, rather than by item number. Manuscripts, in duplicate if possible, may be sent to any one of the four editors. Please classify according to the scheme of Math. Rev. Index to Vol. 39. All other communications to the editors should be addressed to the managing editor, or Elaine Barth, University of California, Los Angeles, California, 90024.

50 reprints are provided free for each article; additional copies may be obtained at cost in multiples of 50 .

The Pacific Journal of Mathematics is issued monthly as of January 1966. Regular subscription rate: $\$ 48.00$ a year (6 Vols., 12 issues). Special rate: $\$ 24.00$ a year to individual members of supporting institutions.

Subscriptions, orders for back numbers, and changes of address should be sent to Pacific Journal of Mathematics, 103 Highland Boulevard, Berkeley, California, 94708.

\section{PUBLISHED BY PACIFIC JOURNAL OF MATHEMATICS, A NON-PROFIT CORPORATION}

Printed at Kokusai Bunken Insatsusha (International Academic Printing Co., Ltd.), 270, 3-chome Totsuka-cho, Shinjuku-ku, Tokyo 160, Japan.

* C. R. DePrima California Institute of Technology, Pasadena, CA 91109, will replace J. Dugundji until August 1974.

Copyright (C) 1973 by

Pacific Journal of Mathematics

All Rights Reserved 


\section{Pacific Journal of Mathematics}

\section{Vol. 49, No. $2 \quad$ June, 1973}

Wm. R. Allaway, On finding the distribution function for an orthogonal polynomial

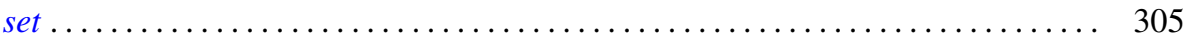

Eric Amar, Sur un théorème de Mooney relatif aux fonctions analytiques bornées... . 311

Robert Morgan Brooks, Analytic structure in the spectrum of a natural system . . . . 315

Bahattin Cengiz, On extremely regular function spaces . . . . . . . . . . . . . . 335

Kwang-nan Chow and Moses Glasner, Atoms on the Royden boundary . . . . . . . . . 339

Paul Frazier Duvall, Jr. and Jim Maxwell, Tame $Z^{2}$-actions on $E^{n} \ldots \ldots \ldots \ldots \ldots . .349$

Allen Roy Freedman, On the additivity theorem for $n$-dimensional asymptotic

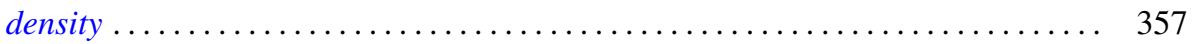

John Griffin and Kelly Denis McKennon, Multipliers and the group $L_{p}$-algebras . . . 365

Charles Lemuel Hagopian, Characterizations of $\lambda$ connected plane continua ....... 371

Jon Craig Helton, Bounds for products of interval functions . . . . . . . . . . . 377

Ikuko Kayashima, On relations between Nörlund and Riesz means . . . . . . . . . . 391

Everett Lee Lady, Slender rings and modules . . . . . . . . . . . . . . . . . . 397

Shozo Matsuura, On the Lu Qi-Keng conjecture and the Bergman representative

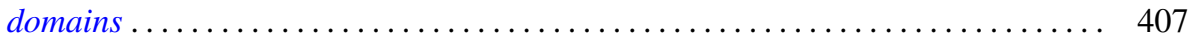

Stephen H. McCleary, The lattice-ordered group of automorphisms of an $\alpha$-set . . . 417

Stephen H. McCleary, o-2-transitive ordered permutation groups .......... 425

Stephen H. McCleary, o-primitive ordered permutation groups. II . . . . . . . . . 431

Richard Rochberg, Almost isometries of Banach spaces and moduli of planar

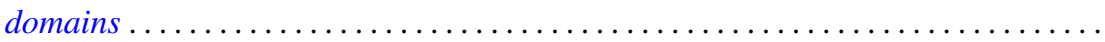

R. F. Rossa, Radical properties involving one-sided ideals . . . . . . . . . . . . . 467

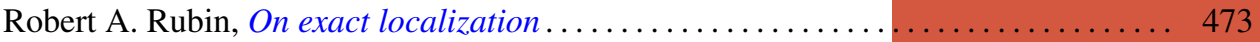

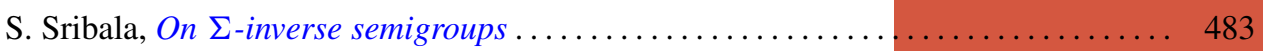

H. M. (Hari Mohan) Srivastava, On the Konhauser sets of biorthogonal polynomials suggested by the Laguerre polynomials ...................... 489

Stuart A. Steinberg, Rings of quotients of rings without nilpotent elements . ...... 493

Daniel Mullane Sunday, The self-equivalences of an $H$-space . . ............ 507

W. J. Thron and Richard Hawks Warren, On the lattice of proximities of $\check{C} e c h$

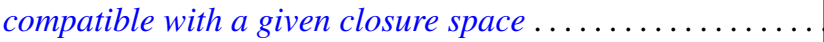

Frank Uhlig, The number of vectors jointly annihilated by two real quadratic forms determines the inertia of matrices in the associated pencil .

Frank Uhlig, On the maximal number of linearly independent real vectors annihilated simultaneously by two real quadratic forms ..............

Frank Uhlig, Definite and semidefinite matrices in a real symmetric matrix pencil . . 561

Arnold Lewis Villone, Self-adjoint extensions of symmetric differential operators . . . 569

Cary Webb, Tensor and direct products . ....................... 579

James Victor Whittaker, On normal subgroups of differentiable

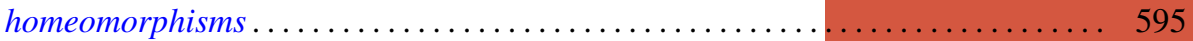

Jerome L. Paul, Addendum to: "Sequences of homeomorphisms which converge to

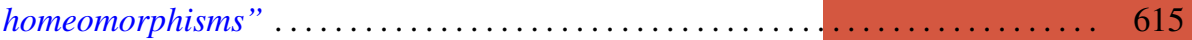

David E. Fields, Correction to: “Dimension theory in power series rings” ........ 616

Peter Michael Curran, Correction to: “Cohomology of finitely presented groups”. . . 617

Billy E. Rhoades, Correction to: “Commutants of some Hausdorff matrices” ...... 617

Charles W. Trigg, Corrections to: "Versum sequences in the binary system” ...... 619 